\title{
Common Source of Cryoprotection and Osmoprotection by Osmolytes
}

\section{Roy Govrin, $¥$ Tal Obstbaum, $₫$ and Uri Sivan \\ Supporting Information}

Department of Physics and the Russell Berrie Nanotechnology Institute, Technion - Israel Institute of Technology, Technion City, Haifa 3200003, Israel

E-mail (Uri Sivan) phsivan@technion.ac.il

$\$$ These authors contributed equally.

Remark regarding temperature control: To facilitate measurements at temperatures lower than $\sim 10{ }^{\circ} \mathrm{C}$, where water vapor would condense on the AFM's optics, the AFM chamber was fed with dry cool air.

\section{Fitting Procedure}

Force measured as a function of surface separation using atomic force microscope (AFM) was fitted by DLVO theory':

$\frac{F(h)}{R}=\frac{Z}{l_{D}} e^{-h / l_{D}}-\frac{H}{6 h^{2}}$,

where the first term on the right-hand side is the electrostatic contribution (double layer repulsion) and the second term is van der Waals contribution. $F$ is the intersurface force measured by AFM, $h$ is the separation between the colloid and surface, $R$ is the colloid radius $(2.5 \mu \mathrm{m}), l_{\mathrm{D}}$ is Debye-Hückel screening length,

$l_{D} \equiv \sqrt{\varepsilon_{0} \varepsilon_{r} k_{\mathrm{B}} T / 2000 N_{A} I e^{2}}$

$H$ is the Hamaker constant (kept constant at $4 \mathrm{pN} \cdot \mathrm{nm}$ ) and

$Z=64 \pi \varepsilon_{0} \varepsilon_{r}\left(k_{B} T / e\right)^{2} \tanh ^{2}\left(e \psi_{0} / 4 k_{B} T\right)$

is the interaction constant. $k_{\mathrm{B}}, T, N_{A}, e, \varepsilon_{r}, \varepsilon_{0}, \psi_{0}, I$ are the Boltzmann constant, the absolute temperature, the Avogadro number, the elementary charge, the relative permittivity, the vacuum permittivity, electrostatic potential at the outer Helmholtz plane ${ }^{2}$ and ionic strength respectively.

Two free parameters, $Z$ and $l_{\mathrm{D}}$, were extracted for each force curve by fitting the data using eq $\mathrm{S} 1$. Surface charge density $(\sigma)$ presented in the main article was then calculated using eq $\mathrm{S} 2$ and

$$
\sigma^{2}=8 \varepsilon_{0} \varepsilon_{r} n k_{B} T \sinh ^{2}\left(e \psi_{0} / 2 k_{B} T\right) \text {. }
$$

Being an effective medium theory, DLVO obviously misses short-range physics originating from non-electrostatic interactions and molecular finite size. However, the long-range electrostatic interaction that serves to extract the overall surface charge should be captured correctly also in the presence of osmolytes. The latter obviously show in macroscopic quantities such as the solution dielectric constant and the Debye-Hückel screening length that enter DLVO theory. These aspects were studied in ref 12 in the main article.

It should be noted that the Debye-Hückel screening length varies little with temperature due to cancelation of the explicit temperature dependence ${ }^{3}$ against the variation in water dielectric constant with temperature (eq S2). Dielectric constant in GB and Pro was obtained from Refs 4 and 5 (below), respectively.

(1) Israelachvili, J. Intermolecular and Surface Forces, 3rd Edition; Academic Press: Burlington, MA, 2011.

(2) Zhao, C.; Ebeling, D.; Siretanu, I.; van den Ende, D.; Mugele, F. Nanoscale 2015, 7 (39), 16298. 
(4) Edsall, J. T.; Wyman, J. J. Am. Chem. Soc. 1935, 57 (10), 1964.

（5） Romano, E.; Suvire, F.; Manzur, M. E.; Wesler, S.; Enriz, R. D.; Molina, M. A. A. J. Mol. Liq. 2oo6, 126 (1-3), 43. 